\title{
Presumed Solitary Dissemination of Colon Cancer Mimicking Primary Cancer of the Small Intestine
}

\author{
Daisuke Inoue Shoji Oura Tomoya Takami Shinichiro Makimoto \\ Department of Surgery, Kishiwada Tokushukai Hospital, Kishiwada, Japan
}

\author{
Keywords \\ Colorectal cancer $\cdot$ Metastatic small intestinal tumor $\cdot$ Solitary dissemination
}

\begin{abstract}
A 69-year-old man with abdominal distention was referred to our hospital. The patient had undergone laparoscopic surgery for his Borrmann type 2 rectal cancer 2 years before. In addition to the re-elevation of serum CEA and CA19-9 levels, computed tomography (CT) showed intestinal dilatation, and positron emission CT showed a presumed tumor with abnormal fluorodeoxyglucose accumulation in the small intestine. We judged the small intestinal dilatation was highly due to the solitary recurrent peritoneal dissemination of rectal cancer and performed laparoscopic evaluation of the abdominal cavity followed by laparoscopic resection of the affected small intestine. The small intestinal tumor resembled the rectal cancer both on macroscopical and microscopical findings, that is, Borrmann type 2 phenotype and adenocarcinoma that was well differentiated in the part that protruded into the small intestinal lumen and whose degree of differentiation gradually decreased toward the serosa. In addition, abrupt disruption of the normal small intestinal epithelium and the lymphocytic aggregation, presumed tumor-infiltrating lymphocytes, just between the tumor and the small intestinal epithelium highly suggested the tumor originating from the colon cancer. The patient recovered uneventfully with marked decrease in tumor marker levels 1 month after the operation but did not receive colon cancer-oriented chemotherapy as adjuvant therapy for his financial reasons. Oncologists should note this type of recurrence to properly treat the patients with recurrent colorectal cancer.
\end{abstract}

\section{Karger $\stackrel{2}{\circ}$}




\section{Introduction}

The most common malignant tumor of the small intestine is neuroendocrine tumor followed by adenocarcinoma, stromal tumor, and lymphoma [1]. Adenocarcinoma of the small intestine accounts for $<0.5 \%$ of all malignant tumors and $<5 \%$ of all gastrointestinal malignancies [2]. Due to its difficult accessibility, compared with gastric and colonic malignancies, malignant tumors in the small intestine are rarely diagnosed in patients unless they develop some kind of symptoms such as abdominal pain, weight loss, nausea, gastrointestinal bleeding, and intestinal obstruction.

Colorectal cancer is one of the most common cancers worldwide. Colorectal cancer can spread to the lymph nodes, liver, lung, and peritoneum. Metastatic foci of colorectal cancer, when resectable, are regarded as a good candidate for surgical intervention to improve quality of life or often to get a cure of the patients with oligometastatic colorectal cancer [3, 4]. Peritoneal dissemination of colorectal cancer, however, is rarely treated with locoregional therapy due to its high probability of multiplicity of disseminated foci and frequent accompaniment of cancerous ascites [5].

Pathological evaluation of primary cancer mainly depends on the presence of noninvasive component in various solid malignancies including the small intestinal cancer. When a solitary disseminating focus affects the small intestinal wall and invades throughout the intestinal wall toward intestinal lumen with the disruption of the epithelium of the small intestine, it is sometimes difficult for oncologists to judge the mass protruding into the intestinal lumen whether to be a primary intestinal tumor or a metastatic tumor of the small intestine. We herein report a case of presumed solitary dissemination of colon cancer mimicking primary cancer of the small intestine.

\section{Case Report}

A 69-year-old man with abdominal distention was referred to our hospital in June 2021. In August 2019, the patient had undergone laparoscopic low anterior resection with lymph node dissection for his Bormann type 2 rectal cancer (Borrmann type 2, adenocarcinoma, pT4a, pN2a, pStage IIIc; Fig. 1a, b, Fig. 2a, b, and Fig. 3a-c) followed by adjuvant chemotherapy. Elevated preoperative carcinoembryonic antigen (CEA) level of $115.9 \mathrm{ng} / \mathrm{mL}$ (normal range; $0-5.0 \mathrm{ng} / \mathrm{mL}$ ) and CA19-9 level of $48 \mathrm{U} / \mathrm{mL}$ (normal range; 0-37 U/mL) had normalized after rectal cancer operation. On the present event, computed tomography (CT) showed intestinal dilatation, and positron emission CT (PET-CT) showed a presumed tumor with a maximum standardized uptake value of 13.5 in the small intestine located in the left lower abdomen (Fig. 1c). There were no other abnormal accumulations in the abdomen and other distant organs. Serum CEA and CA19-9 levels had gradually re-elevated up to $22 \mathrm{ng} / \mathrm{mL}$ and $52 \mathrm{U} / \mathrm{mL}$ on abdominal distention, respectively. We judged the small intestinal dilatation due to the recurrent peritoneal dissemination of rectal cancer and decided to perform laparoscopic evaluation of the abdominal cavity. On laparoscopic inspection, we confirmed no other dissemination and the adhesion of the affected small intestine to the abdominal wall in the left pelvic cavity. After resolving adhesions around the presumed tumor, the affected small intestine was resected with enough safety margins, and functional anastomosis was done to the stumps of the small intestine. Macroscopic findings showed a Borrmann type 2 tumor (Fig. 2c, d), similar to that of primary rectal cancer, measuring $25 \times 20 \mathrm{~mm}$ in size. Pathological study showed atypical cells growing in cribriform and tubular fashion and protruding into the small intestinal lumen without overlying normal small intestinal epithelium, and the degree of differentiation of adenocarcinoma gradually decreased toward the serosa (Fig. 3d-f). These

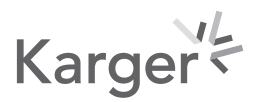




\section{Case Reports in Oncology}
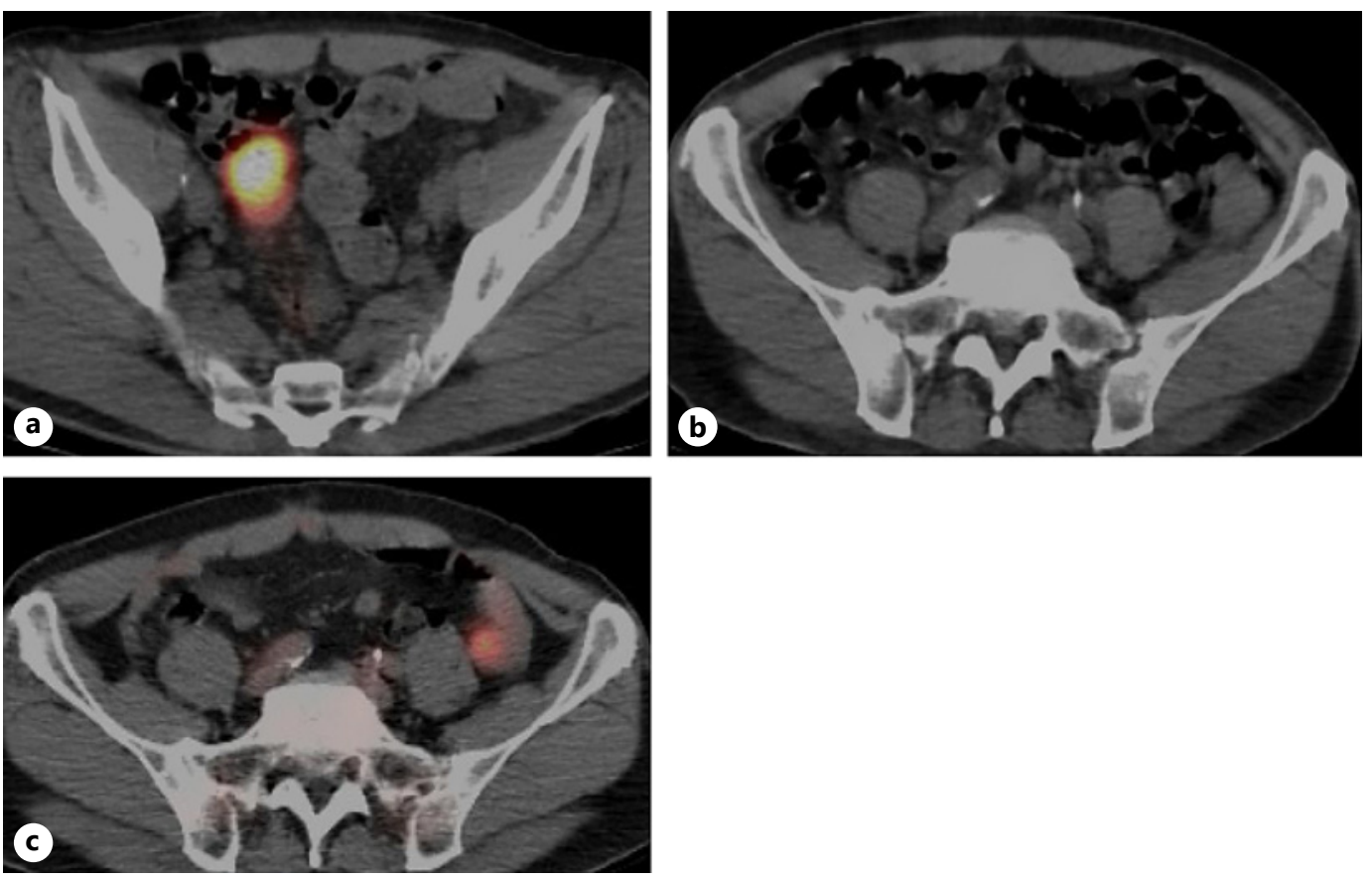

Fig. 1. PET/CT. a PET/CT just before rectal cancer surgery showed marked avidity, that is, a standardized uptake value of 8.9, in the rectosigmoid colon. $\mathbf{b}$ PET/CT on initial surgery showed no accumulations in the small intestine in the left lower abdomen. c PET/CT showed marked avidity, that is, a standardized uptake value of 13.5, in the small intestine. PET/CT, positron emission tomography/computed tomography.

pathological characteristics of the resected tumor were also similar to those of the rectal cancer. The patient recovered uneventfully and was discharged on the 8th day after operation. One month after operation, elevated CEA and CA19 levels decreased to $12.1 \mathrm{ng} / \mathrm{mL}$ and $36.5 \mathrm{U} / \mathrm{mL}$, respectively. The patient should receive colon cancer-oriented adjuvant chemotherapy but did not receive chemotherapy for his financial reasons.

\section{Discussion}

Mechanism of cancer metastasis generally consists of detachment of cancer cells from the proliferating cancer cell clusters, cancer cell invasion to the surrounding host tissue, intravasation of cancer cells into blood or lymphatic vessels, extravasation of cancer cells from the vessels to the distant tissue, and metastatic growth of the migrated cancer cells [6]. Peritoneal dissemination occurs not through this circulating mechanism but through the mechanism of cancer cell exfoliation beyond the serosa to the abdominal cavity, cancer cell implantation, and mass formation mainly on the peritoneum often accompanied by cancerous ascites.

Rectal cancer in this case invaded the serosa and was exposed to the abdominal cavity [7], highly causing the cancer cell exfoliation onto the peritoneum, that is, peritoneal dissemination. Although peritoneal dissemination is very common as a recurrent pattern of colorectal cancer, it usually develops multiple foci and/or accompanying ascites, leading to extremely rare application of locoregional therapy to this type of recurrence. Solitary peritoneal dissemination, however, can be developed when the cancer cells are initially exfoliated to the limited area and successfully form a clinically detectable dissemination. If re-elevation of tumor 

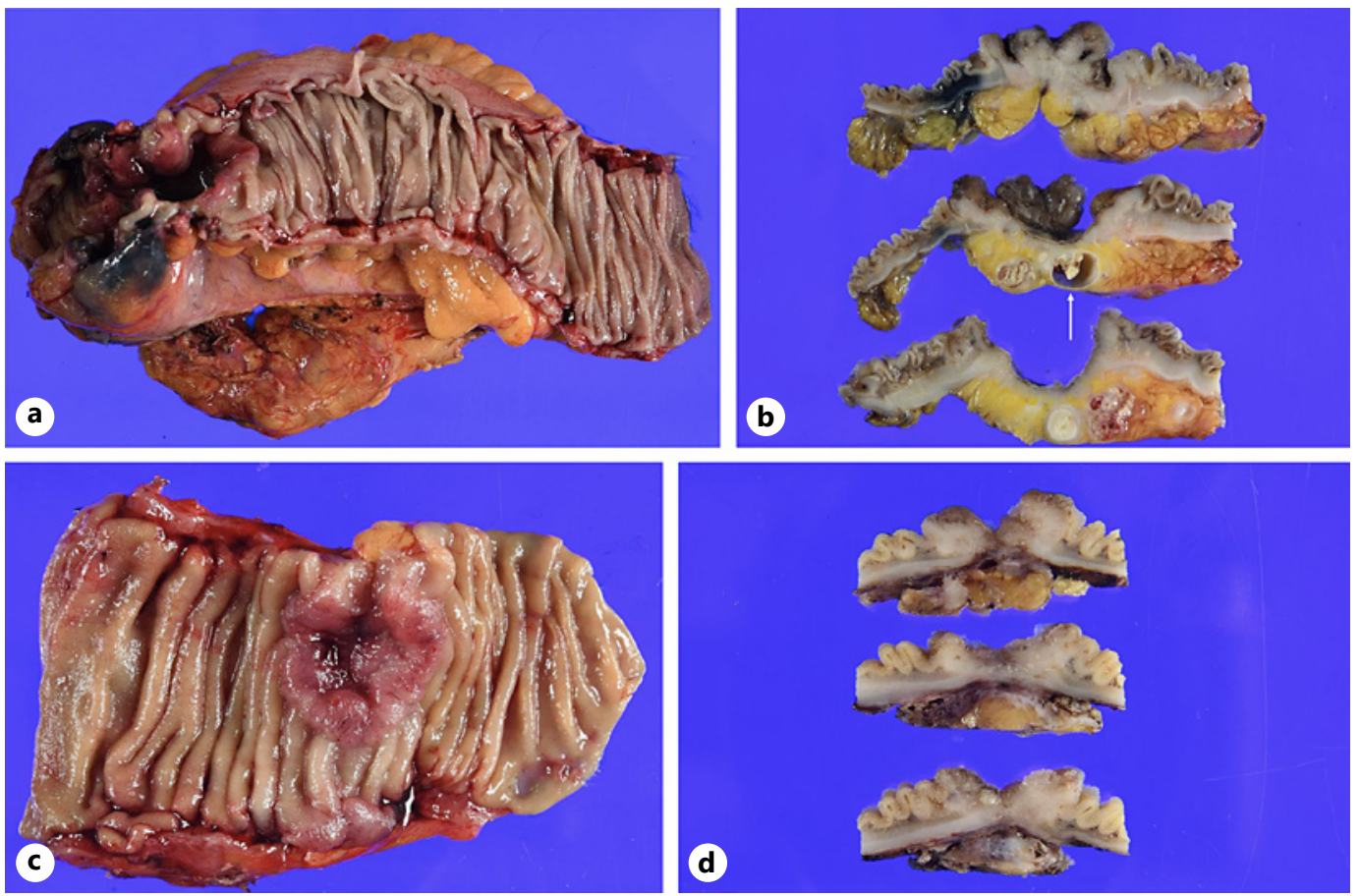

Fig. 2. Macroscopic findings of the rectal cancer and the small intestinal tumor. a Macroscopic findings showed a Borrmann type 2 tumor in the rectum. b Cut surface of the rectal cancer showed a Borrmann type 2 tumor and the distortion of rectal wall with an adjacent cystic lesion (arrow). c Macroscopic findings showed a Borrmann type 2 tumor in the small intestine. $\mathbf{d}$ Cut surface of the tumor showed a Borrmann type 2 tumor similar to that of rectal cancer.

marker levels and abdominal distention had not occurred in this case, we would not have noticed the rectal cancer recurrence until the onset of symptoms caused by cancerous ascites.

The phenomenon that metastatic foci resemble the primary tumor is often experienced. Microscopically speaking, pathological characteristics in the metastatic lymph nodes generally resemble those of primary cancer such as mucinous, signet-ring cell, medullary, micropapillary, serrated, adenoma-like, adenosquamous, and undifferentiated carcinoma [8]. It also is often experienced that configuration of the macroscopic phenotype, that is, enlargement of microscopic properties, resembles that of primary cancer. Shintani et al. [9] reported that Borrmann type 1 gastric cancer developed an intraluminal recurrence after operation, showing Bormann type 1 phenotype similar to that of primary gastric cancer, probably due to cancer cell dissemination through small intestinal lumen to the jejunum close to the anastomotic site.

It was difficult for us to definitely determine the small intestinal focus as a primary small intestinal cancer or a metastatic tumor of the rectal cancer. PET/CT showed no fluorodeoxyglucose accumulations in the small intestine at the time of rectal cancer surgery. CEA levels continued to increase beyond the normal range at and after 14 months postoperatively. We cannot deny the possibility that the primary small intestinal cancer not detected by PET-CT at the time of initial surgery might form a clinically detectable mass in 22 months. Phenotype of the present tumor, however, extremely resembled that of rectal cancer, showing a typical Borrmann type 2 tumor macroscopically and highly differentiated atypical cells protruding into the intestinal lumen, and the degree of differentiation of adenocarcinoma gradually decreased toward the serosa microscopically. In addition, pathological findings showed atypical cells, abrupt disruption of the small intestinal epithelium, and presumed tumor-infil- 


\section{Case Reports in Oncology}

Case Rep Oncol 2021;14:1422-1428
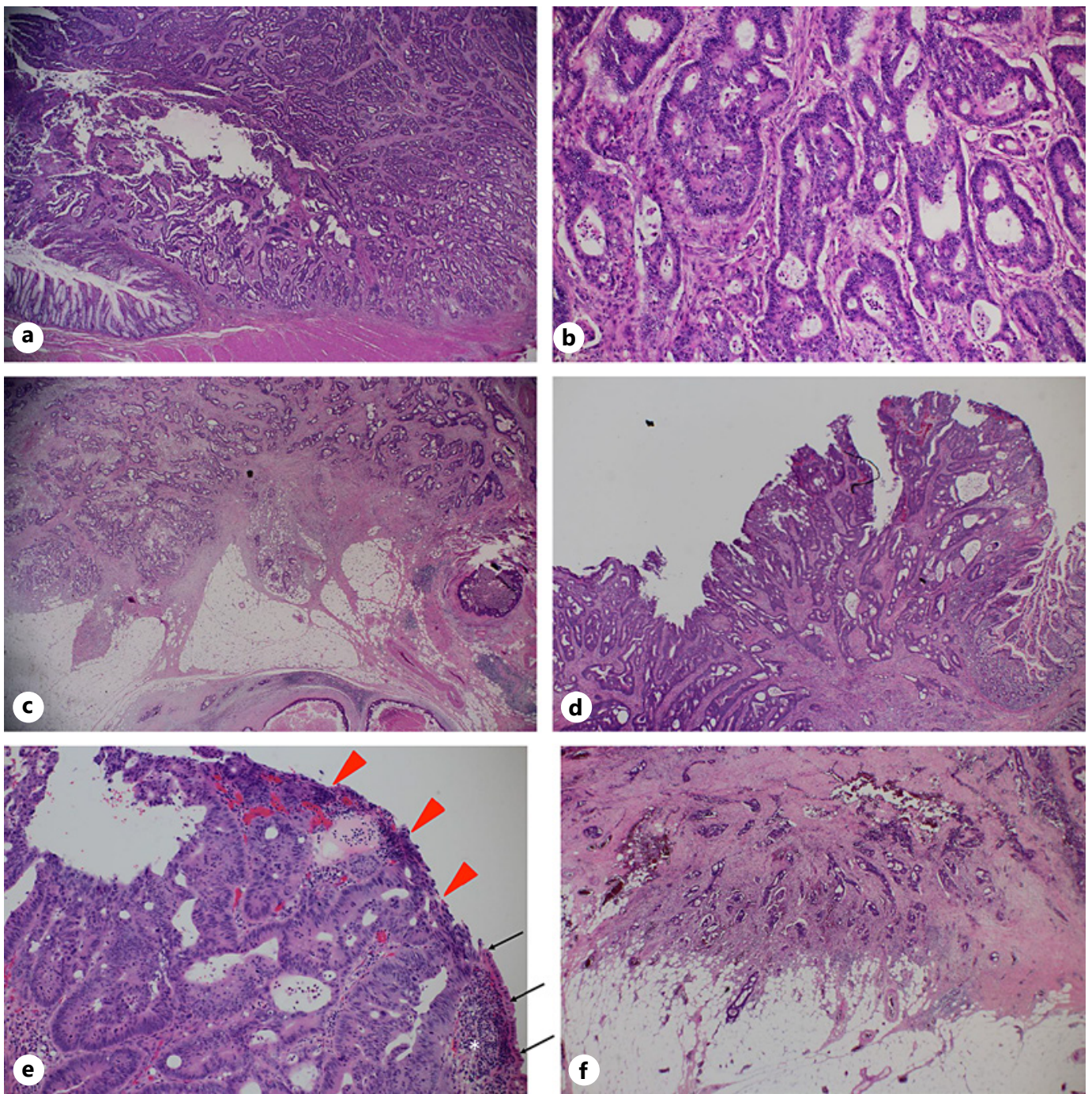

Fig. 3. Pathological findings of the rectal cancer and the small intestinal tumor. a The protruding part of the Borrmann type 2 cancer into the intestinal lumen showed well-differentiated adenocarcinoma growing in a papillary fashion. b Magnified view showed well-differentiated atypical cells. c Atypical cells invading the seroma showed less-differentiated pattern. $\mathbf{d}$ A mass protruding into the small intestinal lumen showed welldifferentiated adenocarcinoma growing in a papillary fashion. e Magnified view showed atypical cells growing in a papillary fashion (arrow heads), intact epithelium of the small intestine (arrows), and presumed tumor-infiltrating lymphocytes (asterisk) just between the atypical cells and the normal epithelium of the small intestine. f Atypical cells showed less-differentiated pattern just around the serosa.

trating lymphocytes located just between the atypical cells and the normal small intestinal epithelium. These findings highly suggest the deciduation of normal small intestinal epithelium, presumably once having existed, due to the atypical cell protrusion toward the intestinal lumen.

All individual tumors initially grow with identical Gompertzian parameters [10]. It, therefore, is more reasonable to consider the present tumor as a recurrence of rectal cancer due to the pathological findings, postoperative changes of tumor marker levels, and very short disease-free interval from the rectal cancer surgery. Adjuvant chemotherapy after macroscopic complete resection of small intestinal cancer has not yet been established, 
suggesting the need for careful observation to this patient without adjuvant chemotherapy. Although we had judged this case as a recurrence of rectal cancer, we unfortunately could not treat the patient with some kind of chemotherapy for his financial reasons.

In conclusion, we experienced a presumed solitary dissemination of colon cancer mimicking primary cancer of the small intestine. Oncologists should be aware of this type of recurrence from colorectal cancer to avoid undertreatment.

\section{Statement of Ethics}

The study is exempt from ethics committee approval due to the nature of reporting based on daily clinical practice. Informed written consent was obtained from the patient for the publication of this case report and any accompanying images.

\section{Conflict of Interest Statement}

The authors have no conflicts of interest to declare.

\section{Funding Sources}

No funding was received for this research.

\section{Author Contributions}

D. Inoue contributed to the design of the report. S. Oura drafted the manuscript. T. Takami treated the patient. S. Makimoto revised the manuscript. All authors have read and approved the final version of the manuscript.

\section{Data Availability Statement}

All data generated during this study are included in this article. Further enquiries can be directed to the corresponding author.

\section{References}

1 Bilimoria KY, Bentrem DJ, Wayne JD, Ko CY, Bennett CL, Talamonti MS. Small bowel cancer in the United States: changes in epidemiology, treatment, and survival over the last 20 years. Ann Surg. 2009;249:63-71.

2 Siegel RL, Miller KD, Jemal A, Jemal A. Cancer statistics, 2017. CA Cancer J Clin. 2021;67:7-30.

3 Tomlinson JS, Jarnagin WR, DeMatteo RP, Fong Y, Kornprat P, Gonen M, et al. Actual 10-year survival after resection of colorectal liver metastases defines cure. J Clin Oncol. 2007;25:4575-80.

4 Booth CM, Nanji S, Wei X, Mackillop WJ. Outcomes of resected colorectal cancer lung metastases in routine clinical practice: a Population-Based Study. Ann Surg Oncol. 2016;23:1057-63.

5 Franko J, Shi Q, Goldman CD, Pockaj BA, Nelson GD, Goldberg RM, et al. Treatment of colorectal peritoneal carcinomatosis with systemic chemotherapy: a pooled analysis of north central cancer treatment group phase III trials N9741 and N9841. J Clin Oncol. 2012;30:263-7.

6 Scully OJ, Bay BH, Yip G, Yu Y. Breast cancer metastasis. Cancer Genomics Proteomics. 2012;9:311-20.

7 Sánchez-Hidalgo JM, Rodríguez-Ortiz L, Arjona-Sánchez Á, Rufián-Peña S, Casado-Adam Á, Cosano-Álvarez A, et al. Colorectal peritoneal metastases: optimal management review. World J Gastroenterol. 2019;25:3484502.

\section{Karger'}


8 Nagtegaal ID, Arends MJ, Salto-Tellez M. Colorectal carcinoma. Digestive system tumours. 5th ed. Genoa: WHO Classification of Tumours; 2019. p. 177-87.

9 Shintani H, Oura S, Makimoto S. Recurrence of gastric cancer in the jejunum close to the anastomotic site after total gastrectomy. Case Rep Oncol. 2021 May-Aug;14(2):767-71.

10 Norton L. A Gompertzian model of human breast cancer growth. Cancer Res. 1988;48:7067-71. 